\title{
Establishing a predictive method for blast induced masonry debris distribution using experimental and numerical methods
}

\author{
Richard A. Keys ${ }^{\mathrm{a}, *}$, Simon K. Clubley ${ }^{\mathrm{a}}$ \\ ${ }^{a}$ Faculty of Engineering and the Environment, University of Southampton, Southampton, UK, SO17 1BJ
}

\begin{abstract}
When subjected to blast loading, fragments ejected by concrete or masonry structures present a number of potential hazards. Airborne fragments pose a high risk of injury and secondary damage, with the resulting debris field causing major obstructions. The capability to predict the spatial distribution of debris of any structure as a function of parameterised blast loads will offer vital assistance to both emergency response and search and rescue operations and aid improvement of preventative measures. This paper proposes a new method to predict the debris distribution produced by masonry structures which are impacted by blast. It is proposed that describing structural geometry as an array of simple modular panels, the overall debris distribution can be predicted based on the distribution of each individual panel. Two experimental trials using $41 \mathrm{~kg}$ TNT equivalent charges, which subjected a total of nine small masonry structures to blast loading, were used to benchmark a computational modelling routine using the Applied Element Method (AEM). The computational spatial distribution presented good agreement with the experimental trials, closely matching breakage patterns, initial fragmentation and ground impact fragmentation. The collapse mechanisms were unpredictable due to the relatively low transmitted impulse; however, the debris distributions produced by AEM models with matching collapse mechanisms showed good agreement with the experimental trials.

Keywords: blast, modular, masonry, fragmentation, debris, applied element method
\end{abstract}

\section{Introduction}

A blast wave is caused by the propagation of a high amplitude shock discontinuity, resulting from physical or chemical detonations [1]. The interaction of blast waves with structures can lead to high levels of structural damage, failure of internal systems, secondary fire, structural collapse, obstructing debris and potentially fatal injury to any occupants [2]. Much research has been conducted into the effects of blast loading and

\footnotetext{
*Corresponding Author: Tel. +44 (0) 2380592862

Email addresses: richakeys@gmail.com (Richard A. Keys), s.k.clubley@soton.ac.uk (Simon K. Clubley)
} 
its interaction with structures, leading to the development of new materials [3], design recommendations $[4,5,6,7]$, comprehensive blast resistant design guides such as UFC-3-340-02 [8] and the handbook for blast resistance design for buildings [9] and the development of pressure-impulse iso-damage curves, illustrated by Figure 1 .

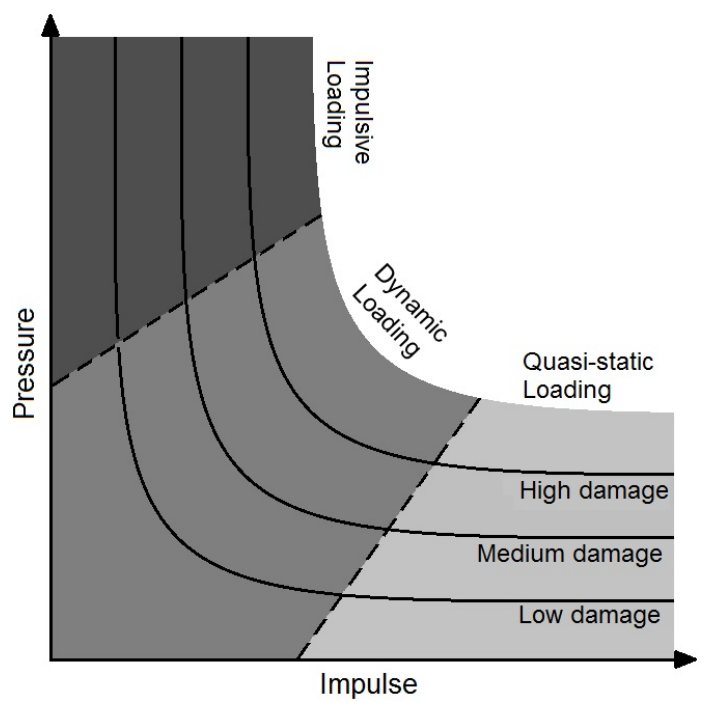

Figure 1: Representation of iso-damage curves

Pressure-impulse iso-damage curves are used to predict damage response and assess the level of damage caused by a specific load and are derived through experimental and numerical investigations [10, 11]. For structures built from primarily brittle materials, such as concrete and masonry, the higher levels of damage result in particulate breakage and fragmentation, especially in the dynamic loading region of the pressureimpulse diagram. Various trials have observed high levels of initial fragmentation when subject to blast loads [12], with near-field detonations producing the highest level of fragmentation and spalling [13, 14]. Many research studies have investigated different aspects of blast induced fragmentation of brittle materials, such as the debris launch velocity $[15,16]$, fragment size [17], ground impact reaction [18] and general statistical descriptions [19].

A spatial debris distribution highlights the areas of high density and the $x, y, z$ extents of the rubble, amongst other aspects, which in turn can provide valuable information to search and rescue operations and even suggest potential preventative measures. This research proposes a new method to predict the area covered by debris resulting from blast loading of masonry structures. The wider objective is to incorporate the model into a fast running engineering tool with the ability to offer accurate predictions of the size, shape and location of the debris field produced by any single story masonry building. 
As the majority of masonry structures are built using walls connected at right angles; such structures can be divided into a set of simplified panels, with unit length $l$, depth $d$ and height $h$, varying in shape and orientation to the incoming blast wave. These modular panels can be arranged in any combination to create a geometrical representation of most buildings, as illustrated by Figure 2. By subjecting individual panels to various blast loads, characteristic debris distributions can be obtained. When combining panels to create larger structures, the characteristic distribution of each individual panel within the structure will change. As part of a larger project, this research aims to develop a method to predict debris distributions based on the number and orientation of simple panels within a structure. This paper presents a set of experimental blast trials and computational models investigating the breakage and debris distribution of simple masonry panels as described by the modular approach.

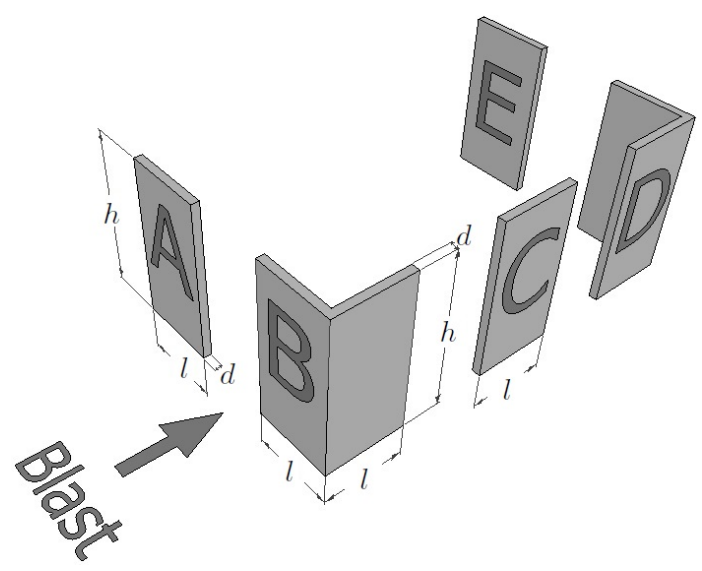

Figure 2: Structure divided into simple panels

\section{Experimental Methodology}

The aim of the experimental trials was to obtain a baseline set of results for the breakage mechanisms and debris distributions of the simple panels, as described by the modular panel approach. The unit length and height of the structures were set to $l=1 \mathrm{~m}$ and $h=2 \mathrm{~m}$ respectively, allowing panels to be constructed with simple integer aspect ratios. Furthermore, these particular dimensions matched those of previous trials conducted by Keys and Clubley [20], allowing earlier experimental results to be incorporated into the data set. To ensure further consistency, the material parameters were also kept consistent with the previous experimental trials. The masonry panels were thus constructed from frogged, facing London bricks with dimensions $210 \mathrm{~mm} \times 100 \mathrm{~mm} \times 65 \mathrm{~mm}$ joined in single leaf running bonds using a class (ii) mortar in a $10 \mathrm{~mm}$ bedding. The bricks had a minimum design compressive strength, $f_{k}$, of $25 \mathrm{MPa}$, with tested compressive 
strengths of between $40-60 \mathrm{MPa}$, with an approximate mass of $2.1 \mathrm{~kg}$. At the time of firing, the maximum compressive strength of the mortar, $f_{m}$, was tested with an average of $7 \mathrm{MPa}$.

Two high explosive experimental blast trials were conducted at the Windrush Arena, MoD Shoeburyness in the UK. Both trials used a 39kg TNT-Flake charge driven by $2 \mathrm{~kg}$ PE4, giving a total TNT equivalence of $41 \mathrm{~kg}$, with structures utilising the panel geometries from Figure 2, in which the unit length, depth and height were fixed at $l=1 \mathrm{~m}, d=0.1 \mathrm{~m}$ and $h=2 \mathrm{~m}$ respectively. The mass of the charge was chosen such that the closest structure would experience an overpressure of approximately $110 \mathrm{kPa}$, whilst remaining outside of the thermal fireball; this particular overpressure also allows additional comparisons to be made with previous research [20].

The trials were designated WR1 and WR2, with full schematics illustrated in Figures 3 and 4 respectively, highlighting the relative positions of each structure, pressure gauge and phantom camera. The first trial, WR1, instrumented five simple flat panels of type A, labelled S1A-S1E and the second trial, designated WR2, instrumented three corner panels of type $\mathbf{B}$, labelled S2A-S2C and one corner panel of type $\mathbf{D}$, labelled S2D. The structures were placed at varying radial distances to achieve a range of target overpressures, calculated using the Kingery and Bulmash polynomials [21]. Two spokes of Endveco-8510 piezoresistive pressure gauges were fielded for each trial, such that incident overpressure at each radial point of interest was monitored by two gauges. Two high speed phantom cameras, capturing at 2,000fps, were positioned to monitor the structure at the nearest radial position from the downstream and side-on perspectives. Finally, to assist with the debris collection, $0.5 \mathrm{~m} \times 0.5 \mathrm{~m}$ grids were drawn around the structures and each brick was given a unique number to identify its original position within the structure.

The full list of radial positions $r$ and corresponding blast parameters, as calculated using the spherical Kingery and Bulmash polynomials [21], are listed in Table 1, where $Z$ is the scaled distance, $p_{i}$ is peak incident overpressure with impulse $I_{i}, p_{r}$ is peak reflected pressure with impulse $I_{r}, t^{+}$is the positive phase duration and $t_{a}$ is the arrival time.

Table 1: Blast parameters at various radial positions for a $41 \mathrm{~kg}$ TNT eq. blast

\begin{tabular}{cccccccc}
\hline \hline$r(\mathrm{~m})$ & $Z\left(\mathrm{mkg}^{-\frac{1}{3}}\right)$ & $p_{i}(\mathrm{kPa})$ & $p_{r}(\mathrm{kPa})$ & $I_{i}(\mathrm{kPa} . \mathrm{ms})$ & $I_{r}(\mathrm{kPa} . \mathrm{ms})$ & $t^{+}(\mathrm{ms})$ & $t_{a}(\mathrm{~ms})$ \\
\hline 10.6 & 3.07 & 110 & 310 & 313 & 752 & 12.8 & 10.0 \\
13 & 3.77 & 72 & 186 & 262 & 595 & 18.1 & 11.4 \\
15 & 4.35 & 55 & 134 & 231 & 506 & 22.9 & 12.3 \\
19.5 & 5.66 & 35 & 79 & 183 & 378 & 34.2 & 13.7 \\
36 & 10.44 & 14 & 29 & 103 & 196 & 78.9 & 16.7 \\
\hline
\end{tabular}




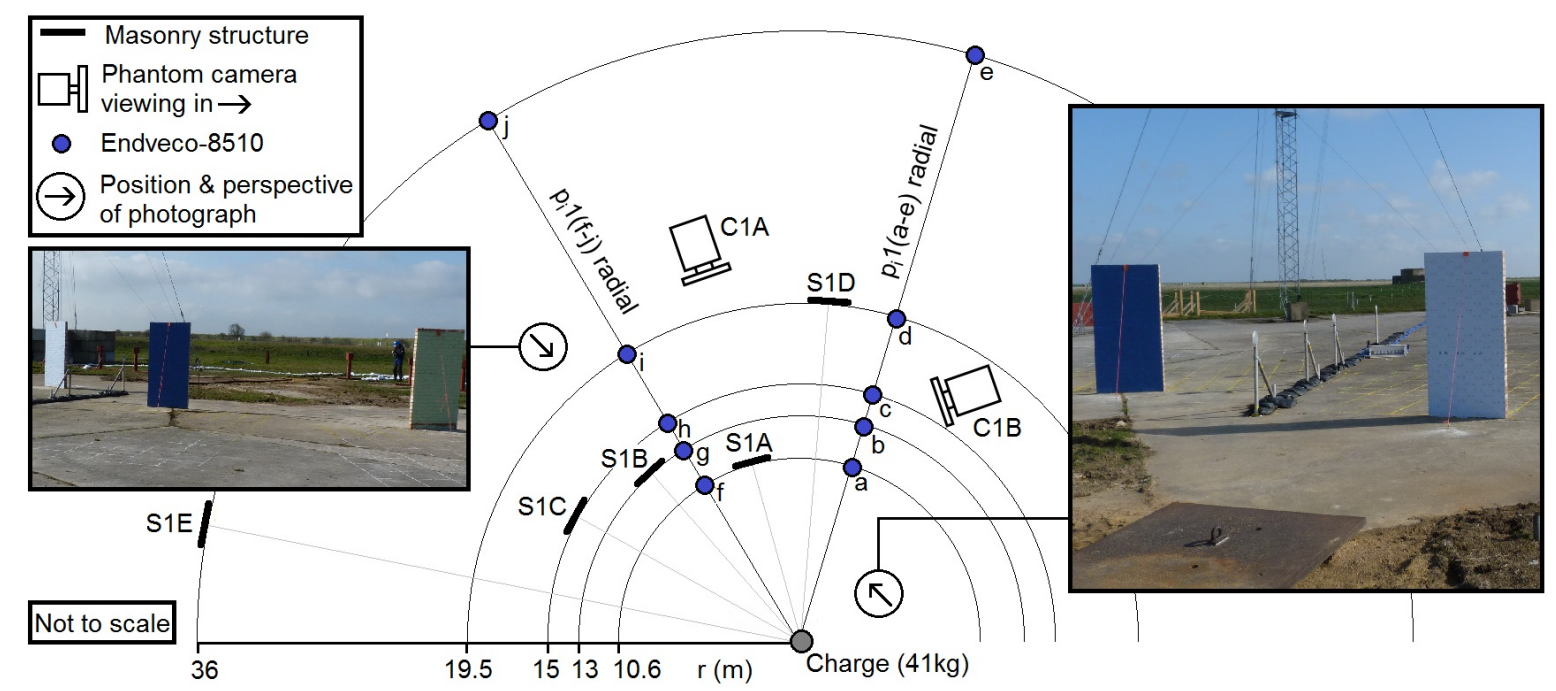

Figure 3: Schematic diagram of trial WR1 from a plan perspective

The $10.6 \mathrm{~m}, 13 \mathrm{~m}$ and $15 \mathrm{~m}$ radials were chosen to achieve a range of higher breakage pressures whilst avoiding the thermal fireball. The first trial, WR1, also featured two lower pressure radials at $19.5 \mathrm{~m}$ and $36 \mathrm{~m}$ populated by structures $\mathrm{S} 1 \mathrm{D}$ and $\mathrm{S} 1 \mathrm{E}$ respectively. The $19.5 \mathrm{~m}$ radial was designed as a boundary case scenario to test the ability of the computational models, as $35 \mathrm{kPa}$ is the approximate limit of masonry breakage. Similarly, structure S1E was designed as a non-responding scenario. Finally, boundary conditions were not applied to any of the structures to simplify the process of comparing individual and composite panel distributions.

\section{Experimental Results}

The pressure-time histories from the trials WR1 and WR2 are displayed in Figures 5a and 5b respectively, with Table 2 highlighting the peak incident overpressure recorded by each pressure gauge and the mean recorded pressures at each radial for each trial. As no structures were implemented at $19.5 \mathrm{~m}$ or $36 \mathrm{~m}$ in trial WR2, data from gauges $p_{i} 2(\mathrm{~d}, \mathrm{e}, \mathrm{i} \& \mathrm{j})$ are not included. The gauge results from the first trial, WR1, showed relatively good agreement between both sets of gauges with a mean difference between the two readings of $16.8 \%$ showing the best agreement with a $12.1 \%$ difference at the $15 \mathrm{~m}$ radial position. The results from the $p_{i} 1(\mathrm{a}-\mathrm{e})$ radial spoke presented the best agreement with the target values, showing a mean difference of $3.9 \%$, whereas the $p_{i} 1(\mathrm{f}-\mathrm{j})$ radial had a mean difference of $13.3 \%$ when compared to the target values. Overall the mean recorded peak pressures from the WR1 trial were between 2.4-8.8\% of the target values.

Results from the $p_{i} 2(\mathrm{a}-\mathrm{c})$ radial spoke from trial WR2 showed good agreement with the target values, 


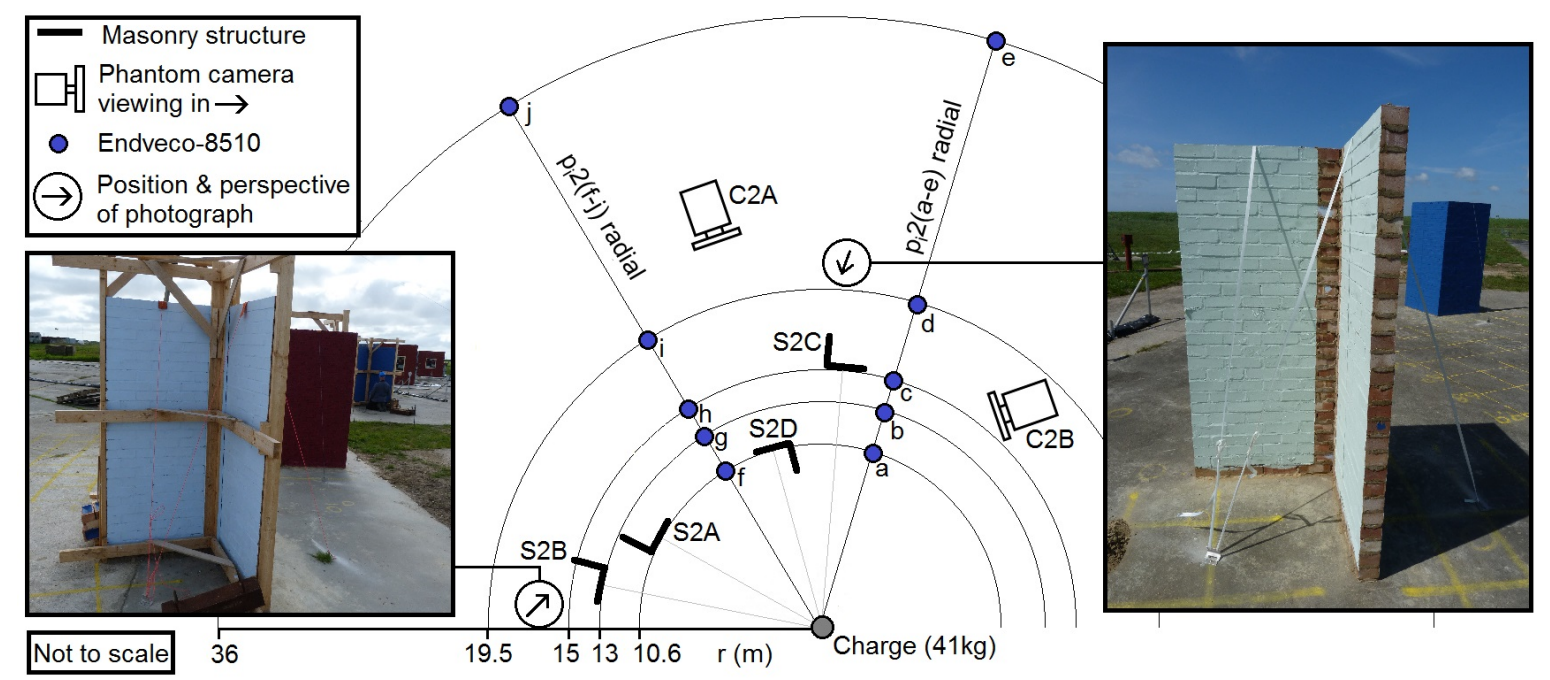

Figure 4: Schematic diagram of trial WR2 from a plan perspective

presenting a mean difference of $9.5 \%$. Gauges $p_{i} 2(\mathrm{f}-\mathrm{h})$ however, specifically gauges $p_{i} 2 \mathrm{f}$ and $p_{i} 2 \mathrm{~g}$ showed large disagreements with both the predicted values and with the corresponding $p_{i} 2 \mathrm{a}$ and $p_{i} 2 \mathrm{~b}$ gauges, likely due to an electrical fault, resulting in a mean difference of $38.9 \%$ with the target values. If these results are separated, then the mean difference in predicted and recorded values across all gauges was $8.3 \%$, with a variance of $8.0 \%$ between all recorded values.

Table 2: Experimental blast parameters

\begin{tabular}{lccccccc}
\hline \hline \multirow{2}{*}{$r(\mathrm{~m})$} & \multirow{2}{*}{ Target $p_{i}(\mathrm{kPa})$} & \multicolumn{3}{c}{ WR1 $p_{i}(\mathrm{kPa})$} & \multicolumn{3}{c}{ WR2 $p_{i}(\mathrm{kPa})$} \\
& & guage(a-e) & guage(f-j) & $\mu$ & guage(a-c) & guage(f-h) & $\mu$ \\
\hline 10.6 & 110 & 115.2 & 99.0 & 107.1 & 119.6 & 69.7 & 94.7 \\
13 & 72 & 73.1 & 58.2 & 65.7 & 65.4 & 15.3 & 40.4 \\
15 & 55 & 53.1 & 59.5 & 56.3 & 49.1 & 55.7 & 52.4 \\
19.5 & 35 & 37.4 & 29.1 & 33.3 & - & - & - \\
36 & 14 & 13.6 & 15.7 & 14.7 & - & - & - \\
\hline
\end{tabular}

From trial WR1, the high speed photography captured the breakage of structure S1A, which developed two horizontal cracks at approximately $10 \mathrm{~ms}$, with the lower forming between the $5^{\text {th }}$ and $6^{\text {th }}$ layers of brick, approximately $0.4 \mathrm{~m}$ above the base and the upper crack forming between the $12^{\text {th }}$ and $13^{\text {th }}$, approximately $0.9 \mathrm{~m}$ above the base. The lower half of the structure began to tilt in the downstream direction about the base, with the upper section of the structure exhibiting a counter tilting motion in the upstream direction about the crack at $0.9 \mathrm{~m}$. Although the time of crack formation for structure S1B cannot be accurately determined 


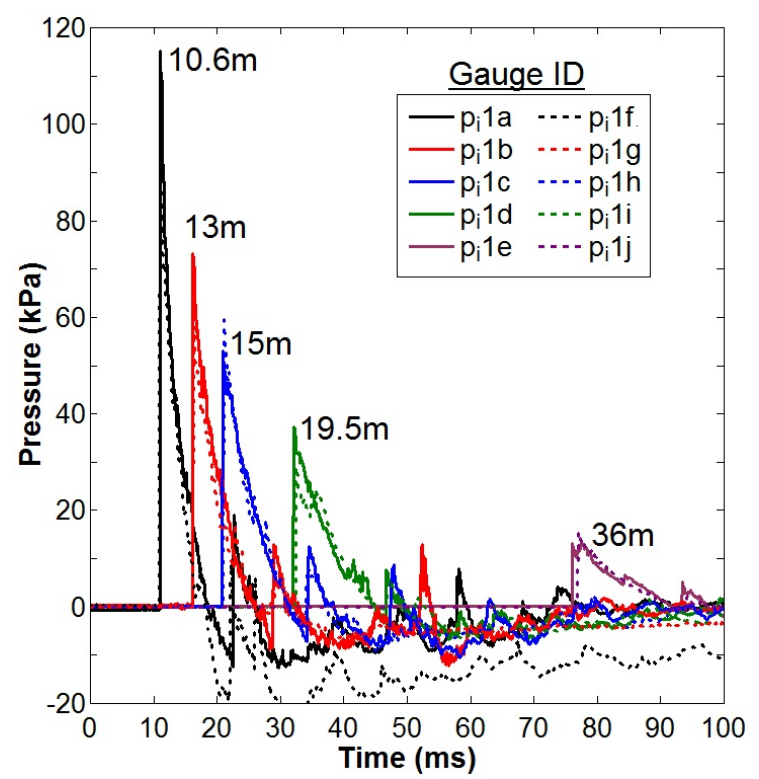

(a) Trial WR1

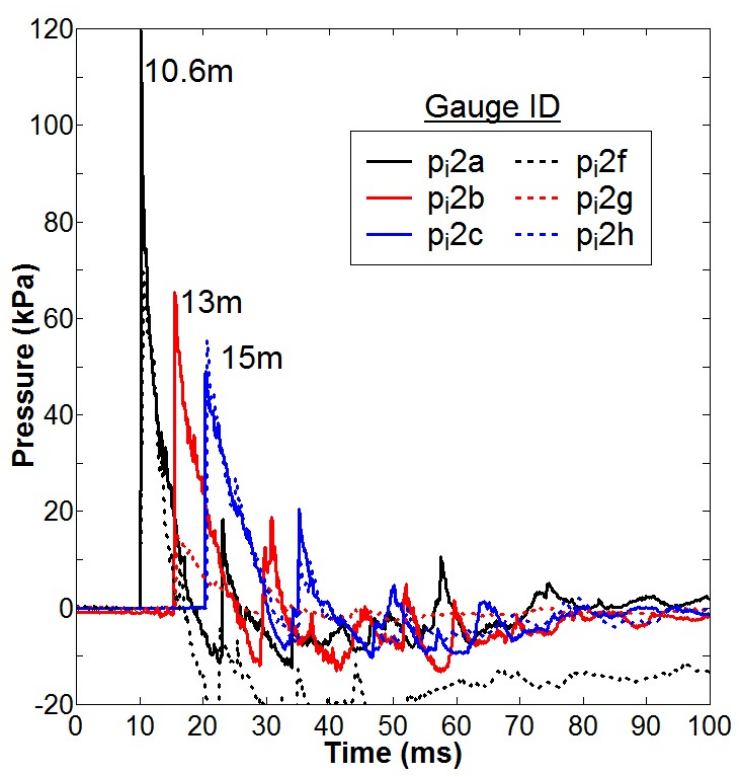

(b) Trial WR2

Figure 5: Incident pressure-time histories from all pressure gauges instrumented in both trials

due to obstructions, a single horizontal crack was observed forming between the $7^{\text {th }}$ and $8^{\text {th }}$ layers of brick, approximately $0.52 \mathrm{~m}$ above the base. The structure exhibited opposing behaviour to structure $\mathrm{S} 1 \mathrm{~A}$, with the lower section tilting in the upstream direction about the base and the upper section tilting in the downstream direction about the base. As no video evidence was recorded, the exact breakage mechanism of structure S1C is unknown, yet the positions of the numbered bricks suggest a crack formed at approximately half height, causing a similar collapse to that of S1A. The boundary case structure located at $19.5 \mathrm{~m}$ remained undamaged showing no signs of breakage; structure S1E, the non-responding scenario at $36 \mathrm{~m}$ also showed no signs of damage as expected.

Whilst the type B corner structure S2A, located at 10.6m, was not the main focus of the high speed photography, partially obscured views from camera C2B suggested that the blast normal panel begin to deflect in the downstream direction by $20 \mathrm{~ms}$. A clear vertical crack formed down the centre of the blast normal face by approximately $40 \mathrm{~ms}$ causing both halves to rotate about the crack in the downstream direction. An additional vertical crack formed along the corner joint which caused the side panel to collapse inwards. Structure S2B, located on the 13m radial, was presented as a boundary case, with a series of vertical, stepped and horizontal cracks forming between $20-80 \mathrm{~ms}$. The structure continued to deflect in the downstream direction about the various cracklines until an equilibrium position was reached at approximately 
200ms. Structure S2B remained in place producing no debris field; however, the extent of the crack left the structure on the brink of collapse, requiring only a small application of force to induce failure. At 15m, structure S2C showed little response; two thin, stepped cracks were observed at the base of the structure along bedding joints and a total of five whole bricks and two half bricks displaced from the upper, outer corner of the blast normal panel. Finally, for type $\mathbf{D}$ structure S2D, the rear facing corner at $10.6 \mathrm{~m}$, showed a network of horizontal and vertical cracks form along the rear, blast normal face after 20ms. A partial, vertical crack at the mid section of the corner joint also formed at approximately 20ms, connected to stepped cracks leading to the centre of the side panel at both the top and bottom of the structure. Horizontal cracks which formed on both faces at approximately $0.5 \mathrm{~m}$ above the base, separated the lower quadrant of the structure, which remained in place. Above this crackline, fragments from the blast normal panel were forced downstream, whilst the side panel tilted outwards due to the internal reflections of the blast wave.

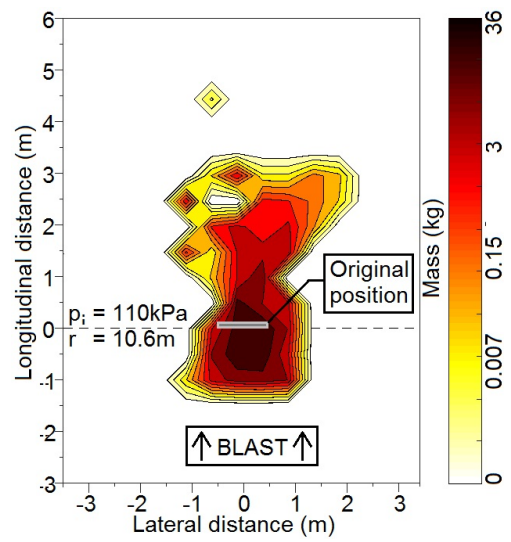

(a) S1A

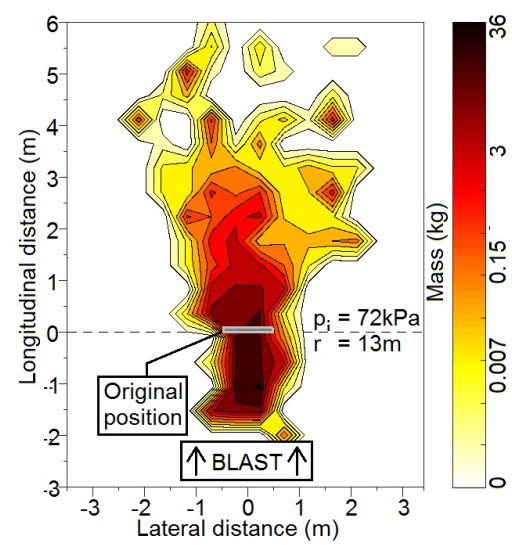

(b) S1B

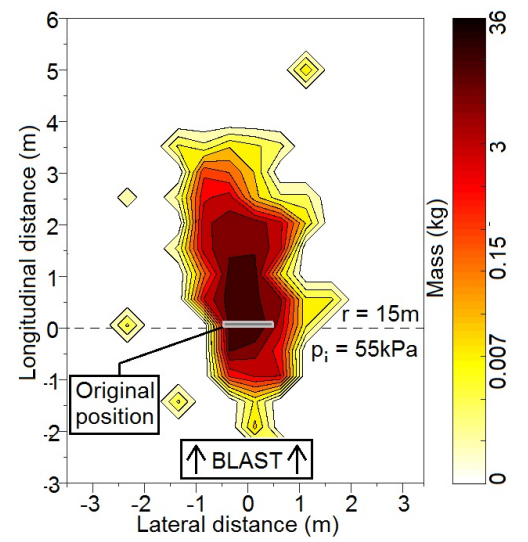

(c) $\mathrm{S} 1 \mathrm{C}$

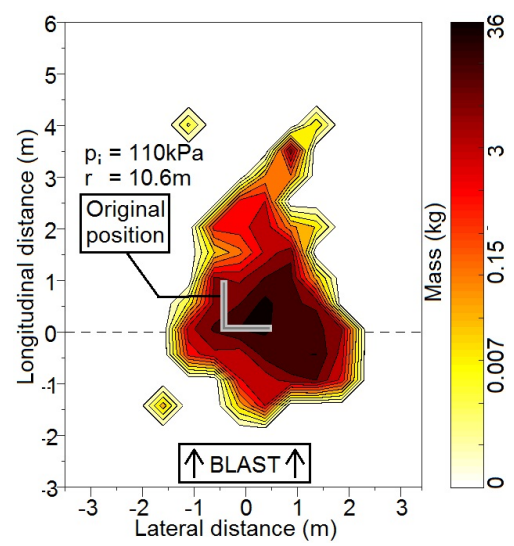

(d) S2A

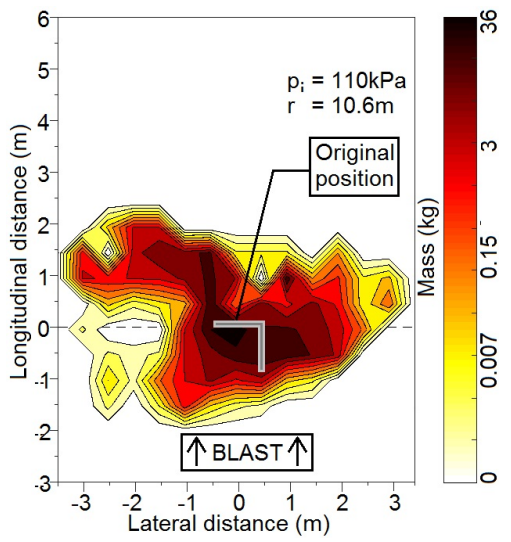

(e) S2D

Figure 6: 2D debris mass distributions of each structure which underwent collapse 
Of the nine structures tested, five structures collapsed and their $x, y$ debris mass distributions are displayed in Figure 6, plotted on a logarithmic scale to indicate the extents of the debris fields including regions of high density. Figures $6 \mathrm{a}, 6 \mathrm{~b}$ and $6 \mathrm{c}$ show the debris distributions of structures S1A (10.6m), S1B $(13 \mathrm{~m})$ and $\mathrm{S} 1 \mathrm{C}(15 \mathrm{~m})$ respectively, indicating the original position of each panel relative to the debris. In each case, a significant portion of the debris is located in the upstream direction due to counter rotation about horizontal crack lines. The largest longitudinal distribution was observed in structure S1B which experienced a peak incident overpressure of $\approx 72 \mathrm{kPa}$. Whilst the highest pressure and impulse is expected to produce the largest distribution, impulse of this particular blast was too low to produce any significant debris throw. As a result the failure mechanisms and hence debris distributions were largely a function of the location of crack formation. For each of the $1 \mathrm{~m} \times 2 \mathrm{~m}$ type $\mathbf{A}$ panels, the lateral distribution was relatively symmetrical about the central vertical axis and contained within $\pm 1 \mathrm{~m}$.

The debris fields produced by structures S2A and S2D, displayed in Figures 6d and 6e respectively, both show high density areas distributed around the original location of the structure. In both cases the lateral debris throw distance was much larger due to collapse of the side panel; however the lateral distribution is considerably larger for the rear facing type $\mathbf{D}$ geometry of structure S2D. As the interior of the structure is exposed to the blast, both panels were forced outwards creating a larger distribution than that of structure S2A. With the blast normal panel at the rear of the structure, the longitudinal distribution of structure S2D is also expected to be larger; however as with the type A panels, the debris distribution is shaped largely by the crack formation for low impulse blast.

Table 3 highlights some statistics from each of the debris distributions, including the approximate area covered $(\approx \mathrm{A})$, the total mass collected $(\Sigma m)$, the mean density $\left(\mu_{\rho}\right)$, the mass of the largest fragment $\left(m_{f}(\max )\right)$, percentage of fragments with mass larger than $0.1 \mathrm{~kg}\left(\Sigma m_{f} \geq 0.1 \mathrm{~kg}\right)$, the percentage of fragments with mass larger than $1 \mathrm{~kg}\left(\Sigma m_{f} \geq 1 \mathrm{~kg}\right)$ and the percentage of undamaged bricks. These statistics show that for each structure the majority of fragments were larger than $1 \mathrm{~kg}$, with between $85-98 \%$ of the bricks remaining undamaged. These statistics also show that the mean area density of the debris is much higher for the corner structures.

\section{Computational Methodology}

Various research studies have used a wide variety of continuous and discretised numerical methods to investigate the response of masonry to a range of loads [22, 23, 24]. Lourenço [25] investigated computational techniques for modelling the behaviour of masonry, particularly micro-modelling, which assumes three main 
Table 3: Experimental debris statistics

\begin{tabular}{cccccccc}
\hline \hline & $\mathrm{A}\left(\mathrm{m}^{2}\right)$ & $\Sigma m(\mathrm{~kg})$ & $\mu_{\rho}\left(\mathrm{kgm}^{-2}\right)$ & $m_{f}(\max )(\mathrm{kg})$ & $\Sigma m_{f} \geq 0.1 \mathrm{~kg}$ & $\Sigma m_{f} \geq 1 \mathrm{~kg}$ & Whole bricks \\
\hline S1A & 11.0 & 366.3 & 33.3 & 30.5 & $99.3 \%$ & $96.3 \%$ & $93.4 \%$ \\
S1B & 21.3 & 360.7 & 17.0 & 32.5 & $99.2 \%$ & $95.1 \%$ & $98.5 \%$ \\
S1C & 12.8 & 385.6 & 30.2 & 18.0 & $99.6 \%$ & $94.4 \%$ & $88.1 \%$ \\
S2A & 12.3 & 740.0 & 60.5 & 20.5 & $99.7 \%$ & $96.8 \%$ & $93.8 \%$ \\
S2D & 17.5 & 731.4 & 41.8 & 87.8 & $99.8 \%$ & $95.6 \%$ & $84.6 \%$ \\
\hline
\end{tabular}

failure mechanisms; crack formation (indistinguishable under both tension and compression), frictional force between the brick-mortar layers and crushing under shear and compression and shows convincing agreement with experimental results. For larger structures, various homogenization techniques have also been explored $[26,27]$ to reduce the memory and time requirements of mirco-modelling approaches on larger structures. A variety of other techniques for modelling masonry failure such as discrete cracking in which cracks manifest as a physical discontinuity between elements, smeared cracking in which cracks are described as elasticity relations, or a combination of both as presented by Borst et al [28], are popular methods for modelling masonry failure under blast loading as the macro-level failure is determined by the formation of cracks on the micro-scale.

For this project, the Applied Element Method (AEM) [29] was selected, due to its ability to reliably model progressive collapse and the large displacements associated with debris distribution [30, 31]. AEM is a discretised model in which the internal forces are modelled by shear and normal springs which connect neighbouring elements along the faces. The rotational stiffness is determined as a function of the number of springs and is shown to converge quickly, with 10 springs showing a $1 \%$ error when compared to theoretical calculations [29]. AEM is particularly suited to modelling debris distribution as temporary springs are created between elements in close proximity post breakage, which greatly improves the downstream debris predictions. The software Extreme Loading for Structures ${ }^{\circledR}$ which utilises the Applied Element Method was used to model the experimental trials; however initial models showed the inbuilt blast loading feature overestimates blast pressures and impulses based on the Kingery \& Bulmash polynomials [21] and does not account for wrap around pressure or clearing effects. For this reason, the Computational Fluid Dynamics (CFD) software Air3D v9 [32] was used to determine the reflected blast pressure across the entire surface of the structure.

Initial domain and mesh size sensitivity studies were conducted to compare the incident pressure time histories produced by Air3D to those recorded experimentally to assess the accuracy and reliability of the 
results. For all models, the blast wave is initially propagated in 1D from the charge location to the edge of the domain, where it is remapped into 3D. The domain size was iteratively reduced from a $10 \mathrm{~m}^{2}$ radius centered around the structure to approximately $5 \mathrm{~m}^{2}$ in both the upstream and downstream directions and 4 $\mathrm{m}^{2}$ in both lateral and vertical directions. This ensured the entire blast wave was able to propagate on either side of the structure, whilst maintaining enough distance from the domain boundaries, such that distortions in the curvature of the blast wave have no effect on the centre of the domain during run time. The mesh density was also increased, with a reduction in cell size from $150 \mathrm{~mm}$ down to $40 \mathrm{~mm}$. When comparing the overpressure produced at the relevant stand-off distances from both Air3D and the experimental guage data, there was little gain in accuracy beyond this threshold. Furthermore, this resolution provides coverage of 2500 cells for a $1 \mathrm{~m} \times 2 \mathrm{~m}$ panel, providing high resolution load data to feed into the AEM models.

Figure 7a compares the incident overpressure time histories recorded from gauges $\mathrm{p}_{i} 1(\mathrm{~A}-\mathrm{E})$ to those obtained through Air3D using a cell size of $40 \mathrm{~mm}$ within a domain of between $5.13 \times 10^{6}$ and $7.88 \times 10^{6}$ cells, depending on the distance from the blast and hence blast wavelength. The arrival times have been adjusted, allowing for easy visual comparison. Overall the Air3D results show good agreement between peak overpressure, blast duration, decay and impulse as displayed in Figure 7a. With confidence in the CFD results, the reflected pressure across the surface of the structures were assessed by 325 uniformly distributed monitoring points placed on each face of the structure. Using the results obtained from the monitoring points, both the net pressure and net impulsive loading regions of the structure can be examined by subtracting the rear load curve from front load curve for corresponding monitoring points. The resulting pressure and impulse regions for type $\mathbf{A}$ structure S1A are displayed in Figures 7b and 7c respectively.

With a positive phase duration of approximately $10 \mathrm{~ms}$, the entire impulse was transferred to the structure before the initial crack lines developed at 20ms, suggesting that in this case, the impulsive loading regions dictate the breakage pattern. The load curves were averaged over regions of high, medium and low cumulative impulse according to Figure 7c and applied to the AEM model. The AEM models used bricks formed of single elements based on the high percentage of undamaged bricks observed from each of the trials, outlined by Table 3 and the mortar was modelled explicitly using element $x, y, z$ dimensions of $20 \mathrm{~mm} \times 20 \mathrm{~mm} \times 10 \mathrm{~mm}$. The material parameters were set to match the design specifications of the materials used for testing and kept constant for each model. Finally, the loading was applied with a time step of $0.25 \mathrm{~ms}$, for a total of $3 \mathrm{~s}$ to allow for all fragments to come to rest. 


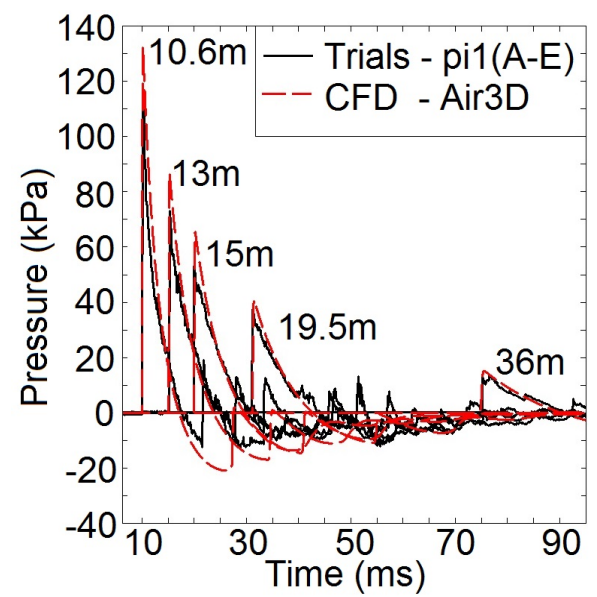

(a) Incident pressure vs WR $1 \mathrm{p}_{i} 1(\mathrm{~A}-\mathrm{E})$

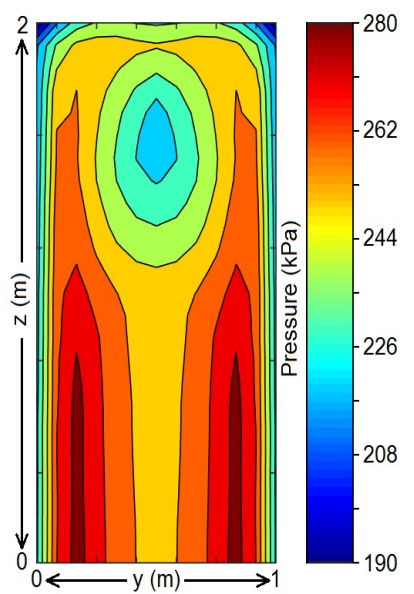

(b) Net peak reflected pressure

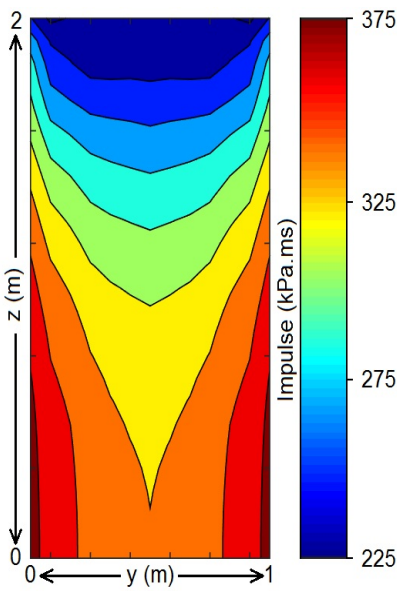

(c) Net impulse

Figure 7: Air3D callibration and loading regions

\section{Computational Results}

The breakage patterns observed from each of the AEM models showed good agreement with those observed in the experimental trials. As with the experimental results, the outlying type A structures, S1D and S1E showed no response, remaining undamaged. The remaining type $\mathbf{A}$ structures each developed horizontal breakage lines within the lower quadrant at approximately $20 \mathrm{~ms}$, closely matching the breakage observed in the trials. Following the crack formation, the structures each tilted about the base and crack lines leading to collapse. The direction of tilt about these cracklines differed when compared to the experimental trials, suggesting the pressure transmitted by the blast wave dictated the location of the crack formation; however the impulse was not high enough to force collapse in the downstream direction. The compressive strength of the mortar for the AEM model of structure S1A was altered in $1 \%$ intervals within $\pm 5 \%$ of the experimental value to investigate the sensitivity of the collapse mechanism. The direction of rotation about the crack lines varied, showing both upstream and downstream tilt about the base for both higher and lower values of the compressive strength. These results suggest that at these threshold impulse values, the direction of tilt is dictated by uncontrollable environmental variables, for example, the mortar application and density of the AEM model is uniform, which is almost impossible to achieve in an experimental structure. Furthermore, additional imperfections such as microfractures and pre-stresses in the experimental structure could not be determined and thus could not be replicated in the AEM models.

Comparisons of the experimental and computationally modelled collapse of structure S1A, displayed in Figures $8 \mathrm{a}$ and $8 \mathrm{~b}$, show the AEM model correctly matching the location of the horizontal crack lines. 
Figures $8 \mathrm{c}$ and $8 \mathrm{~d}$ show the AEM model developing similar rotational moments to the experimental structure, tilting in the downstream direction about its base. The tilting about the upper crack line became more pronounced in the experimental trial, causing the upper section of the structure to tilt in the upstream direction; however the rotational moment in the AEM model was lower, resulting in the upper section of the structure producing a more confined debris distribution.

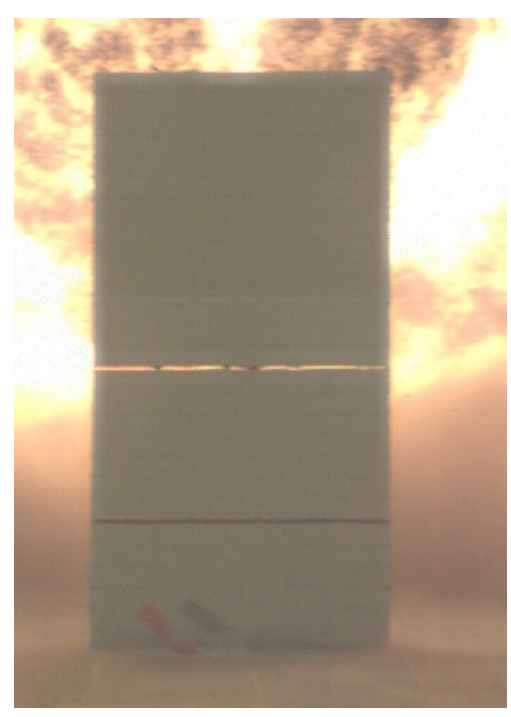

(a) Trial (front)

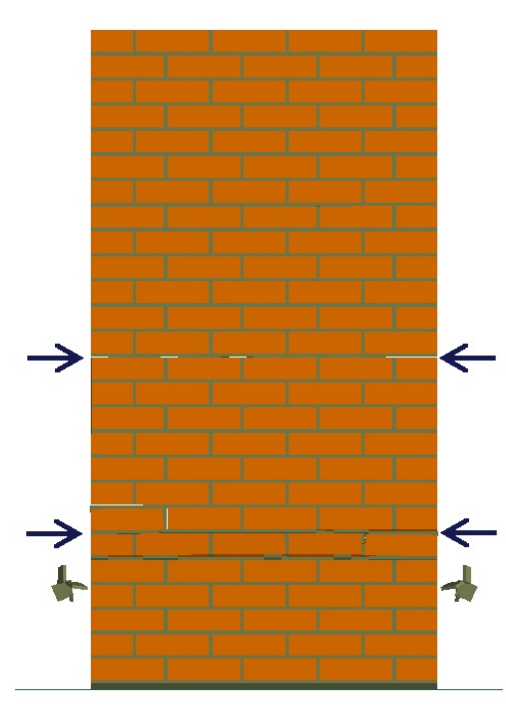

(b) AEM (front)

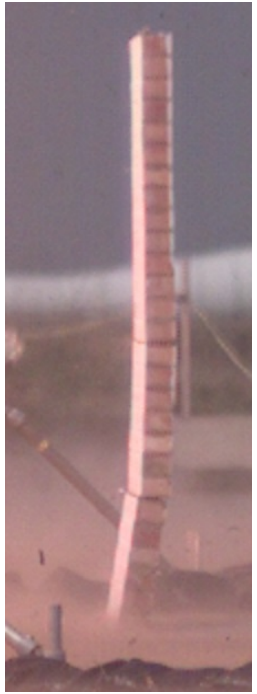

(c) Trial (side)

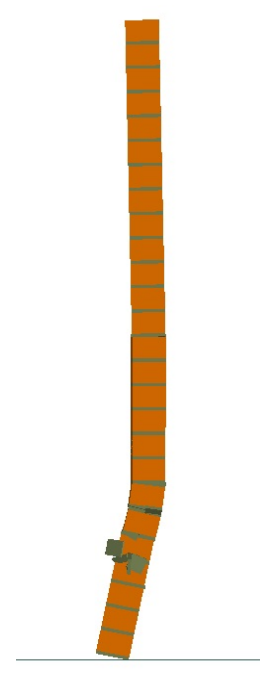

(d) AEM (side)

Figure 8: Breakage of structure S1A at 50ms, computational and experimental comparison

The type $\mathbf{B}$ and $\mathbf{D}$ corner structures exhibited higher levels of initial fragmentation, with several horizontal, vertical and stepped cracks forming across both faces. Structures S2A and S2D, subject to 110kPa, both displayed extensive cracking followed by structural collapse, as observed in the experimental trials. The high speed photography was angled directly at structure S2D, thus offering a more detailed comparison with the AEM model, presented in Figures 9a and 9b. Both the trial photography and AEM model show a horizontal crack form at approximately half height on the side panel, in combination with the blast normal panel collapsing in the downstream direction whilst rotating about the corner joint. The experimental structure showed additional cracking on the side panel, including a vertical crack along joint and a stepped crack running up and out to the edge of the structure. The AEM model did not develop a stepped crack, but did however display increased separation along the vertical crack region due to the single element nature of the bricks, which restrict the formation of clean vertical cracks.

Structure S2B, $72 \mathrm{kPa}$, displayed high levels of damage with extensive cracking in the trials with no 
collapse as displayed in Figure 9c. The AEM model displayed good agreement with the general cracking pattern and resulting deformations, illustrated in Figure 9d; however, the AEM model only retained this unstable position for approximately 1000ms, after which the structure slowly collapsed. This particular result highlights the success of AEM at modelling breakage patterns, temporarily achieving the delicate equilibrium resulting from a boundary case load. The AEM model of the final type $\mathbf{B}$ structure, S2C at $55 \mathrm{kPa}$, showed no response.

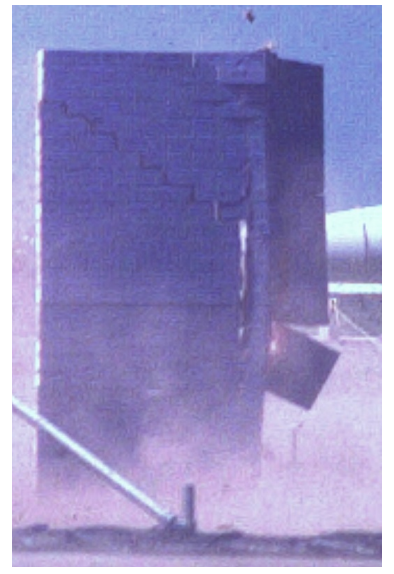

(a) S2D Trial (250ms), blast (b) S2D AEM (250ms), blast left to right

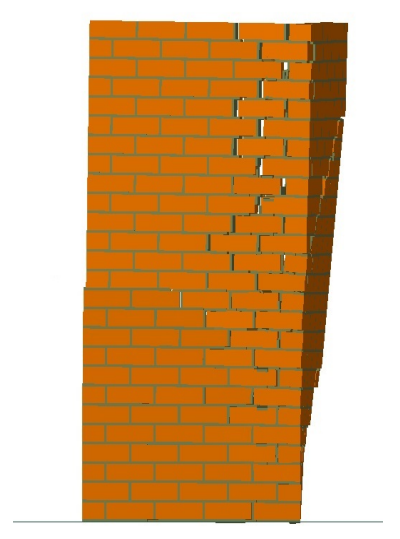
left to right

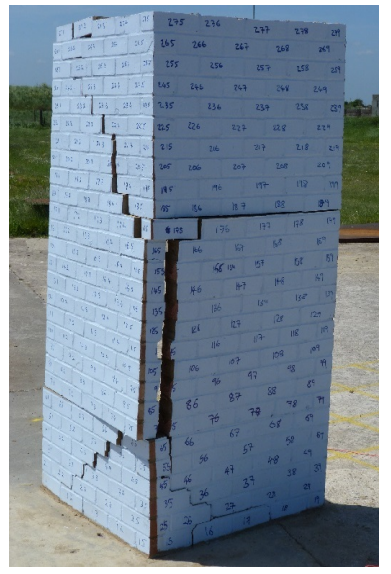

(c) S2B Trial (final)

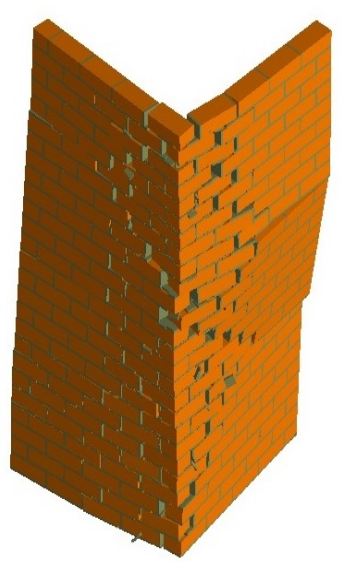

(d) S2B AEM (400ms)

Figure 9: Computational and experimental comparison of structures S2D and S2B

The longitudinal debris distributions of each of the structures which exhibited a response are displayed in Figure 10, comparing the experimental and AEM distributions. In the case of structures S1A and $\mathrm{S} 1 \mathrm{~B}$, with overpressures of $110 \mathrm{kPa}$ and $72 \mathrm{kPa}$ respectively, the AEM results showed a more constricted longitudinal distribution, with high density distributions confined between $0 \mathrm{~m}<x<2 \mathrm{~m}$. For both S1A and $\mathrm{S} 1 \mathrm{~B}$, the experimental results showed a larger portion of the debris located between $-1 \mathrm{~m}<x<0 \mathrm{~m}$. Although the breakage patterns and initial crack formation observed in both structures was similar in both the AEM models and the trials, the direction of tilt about the crack lines differed, resulting in different debris distributions. The AEM debris distribution of structure $\mathrm{S} 1 \mathrm{C}, 72 \mathrm{kPa}$, shared a similar profile with that of the experimental trials. As with the previous type A panels, the cracking patterns observed in both the AEM model and the experimental trial showed good agreement; however, the direction of tilt and hence the collapse mechanism in this particular model matched that of the trial, thus leading to similar distributions.

The AEM debris distributions of the type B corner geometries of structures S2A and S2B both showed good agreement with the experimental results. The distribution of structure S2A in particular shows only 


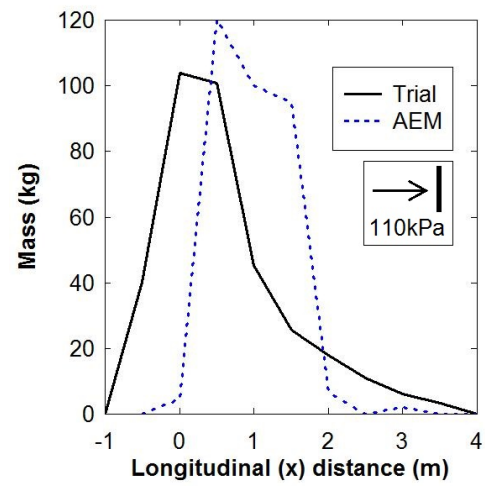

(a) S1A

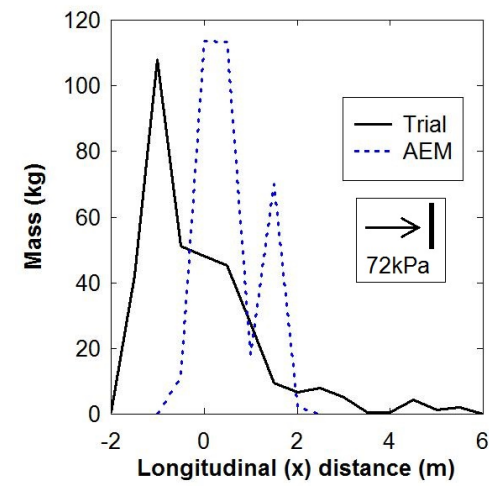

(b) S1B

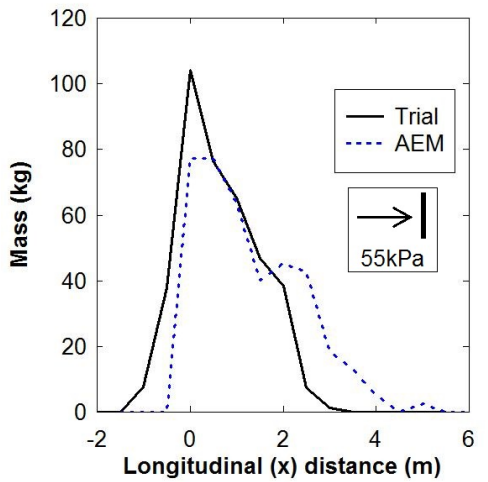

(c) $\mathrm{S} 1 \mathrm{C}$

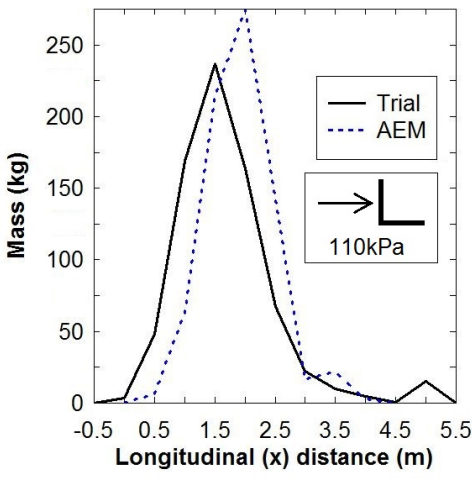

(d) S2A

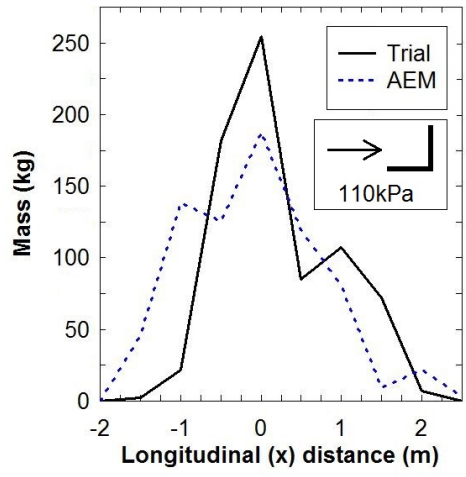

(e) S2D

Figure 10: Longitudinal debris distribution comparisons of AEM models and trials

a $0.5 \mathrm{~m}$ translation in the predicted and observed debris fields, with $\approx 95 \%$ and $\approx 93 \%$ of the debris located between $0 \mathrm{~m}<x<3 \mathrm{~m}$, for the AEM model and trial respectively. For both the trial and AEM model, the debris from structure S2D was located between $-2 \mathrm{~m}<x<2.5 \mathrm{~m}$, with the experimental results showing higher density around the origin and the AEM results showing higher density in the upstream direction. For both type $\mathbf{B}$ structures, the collapse mechanism is more constricted than with the unrestrained type $\mathbf{A}$ panels, due to the additional panel restricting the direction of collapse, thus resulting in better agreement between the distributions. Crack formation along the corner joint and horizontally along the blast normal panel caused the upper section of the blast normal panel to become completely detached from the rest of the structure. This unrestrained fragment is then free to collapse in either direction, leading to the different density regions observed in the distributions of structure S2D. 


\section{Summary \& Conclusions}

This paper proposes a new method to predict the spatial debris distribution produced by masonry panels. Two experimental blast trials were conducted, subjecting a total of 9 unrestrained masonry panels with flat and corner geometries to varying overpressures to assess the effects of geometry on debris distribution. The experimental trials showed the overpressures were high enough to cause breakage, whilst the impulse was too low to produce a replicable collapse mechanism for the flat, $1 \mathrm{~m} \times 2 \mathrm{~m}$ A panels.

The AEM models showed good agreement in terms of crack formation and initial fragmentation for both panel geometries. The AEM models of the type B corner geometries showed good agreement with the experimental debris distributions. Furthermore, the debris distribution of the type A structure S1C showed good agreement with the experimental trial, as a result of the matching collapse mechanism. Based on these results, AEM is expected to offer increased performance for slightly higher impulses, leading to replicable collapse mechanisms.

The proposed modular panel approach potentially offers the ability for fast predictions of masonry debris distribution. The experimental trials offered valuable data to benchmark the AEM modelling routine, allowing further geometries and panel combinations to be tested. Based on these results, larger blast impulses would be required to produce repeatable and reliable debris distributions. Whilst additional experimental results would be favourable, due to the expensive and exclusive nature of blast trials, an extensive parametric study utilising AEM could be used to further develop the modular approach. Data obtained through both experimental and parametric studies will be used to develop a fast running predictive tool to aid emergency response operations.

\section{Acknowledgments}

Funding for analytical research has been gratefully provided by EPSRC Grant: EP/M009254/1. The authors would like to express gratitude to the UK Ministry of Defense for providing the use of testing facilities. All data hereby obtained through the use of such facilities remains the property of the UK MoD.

\section{References}

[1] B. Gelfand, M. Silnikov, Blast Effects Caused by Explosions, European Research Office of the U.S. Army, 2004.

[2] H. Draganic, V. Sigmund, Blast loading on structures, Technical Gazette 19 (3) (2012) 643 - 652.

[3] B. Cavili, M. Rebentrost, Ductal - a high performance material for resistance to blasts and impacts, Australian Journal for Structural Engineering 7 (1) (2006) $37-45$. 
[4] M. D. Goel, V. A. Matsagar, Blast-resistant design of structures, Practice Periodical on Structural Design and Construction $19(2)$.

[5] H. S. Norville, E. J. Conrath, Blast-resistant glazing design, Journal of Architectural Engineering 12 (3) (2006) 129 - 136.

[6] Z. Koccaz, F. Sutcu, N. Torunbalci, Architectural and structural design for blast resistant buildings, in: 14th World Conference on Earthquake Engineering, Beijing, China, 2008, pp. $1-8$.

[7] R. Codina, D. Ambrisubu, F. de Borbn, Alternatives to prevent the failure of rc members under close-in blast loadings, Engineering Failure Analysis 60 (2016) 96 - 106.

[8] Unified Facilities Criteria (UFC), Structures to resist the effects of accidental explosions (ufc 3-340-02), Tech. rep., United States Departement of Defence (December 2008).

[9] D. O. Dusenberry (Ed.), Handbook for Blast-Resistant Design of Buildings, Wiley, 2010.

[10] R. N. Schumacher, B. E. Cummings, A modified pressure-impulse blast damage model, Tech. rep., U.S. Army Ballistics Research Laboratory (January 1977).

[11] F. Parisi, C. Balestrieri, D. Asprone, Blast resistance of tuff stone masonry walls, Engineering Structures 113 (2016) 233 -244 .

[12] R. A. Keys, S. K. Clubley, Modelling debris distribution of masonry panels subject to blast loads using experimental \& applied element methods, in: 15th International Symposium on Interaction of the Effects of Munitions with Structures, Potsdam, Germany, 2013, pp. 1 - 10.

[13] S. jian Yao, D. Zhang, F. yun Lu, W. Wang, X. guang Chen, Damage features and dynamic response of rc beams under blast, Engineering Failure Analysis 62 (2016) 103 - 111.

[14] A. Doerr, N. Gebbeken, M. Larcher, M. Steyerer, C. Haberacker, The effect of near-field explosions on masonry walls, in: 15th International Symposium on the Interactions and Effects of Munitions and Structures, Potsdam, Germany, 2013, pp. $1-12$.

[15] K. Xu, Y. Lu, Debris velocity of concrete structures subject to explosive loading, International Journal for Numerical and Analytical Methods in Geomechanics 30 (2006) 917 - 926.

[16] Y. Lu, K. Xu, Prediction of debris launch velocity of vented concrete structures under internal blast, International Journal of Impact Engineering 34 (2007) 1753 - 1767.

[17] M. Wang, H. Hao, Y. Ding, Z.-X. Li, Prediction of fragment size and ejection distance of masonry wall under blast load using homogenized masonry material properties, International Journal of Impact Engineering 36 (2009) 808 - 820.

[18] C. Knock, I. Horsfall, S. M. Champion, I. C. Harrod, The bounce and roll of masonry debris, International Journal of Impact Engineering 30 (2004) $1-16$.

[19] M. M. van der Voort, J. Weeheijm, A statistical description of explosion produced debris dispersion, International Journal of Impact Engineering 59 (2013) $29-37$.

[20] R. A. Keys, S. K. Clubley, Experimental analysis of small masonry panels subject to long duration blast loading, in: 16th International Symposium on Interaction of the Effects of Munitions with Structures, Sandestin, Florida, 2015 , pp. 1 - 7.

[21] C. N. Kingery, G. Bulmash, Airblast parameters from tnt spherical air burst and hemispherical surface burst, Tech. rep., U.S. Army Armament Research and Development Center, Ballistic Research Laboratory, Maryland (1984).

[22] A. Alexandris, E. Protopapa, I. Psycharis, Collapse mechanisms of masonry buildings derived by the distinct element method, in: 13th World Conference on Earthquake Engineering, Vancouver, Canada, 2004, pp. 1 - 14.

[23] H. Hao, G.-W. Ma, Y. Lu, Damage assesssment of masonry infilled rc frames subjected to blasting induced ground 
excitations, Engineering Structures 24 (6) (2002) $799-809$.

[24] S. Noor-E-Khuda, M. Dhanasekar, D. P. Thambiratnam, An explicit finite element modelling method for masonry walls under out-of-plane loading, Engineering Structures 113 (2016) 103 - 120.

[25] P. B. Lourenço, Computational strategies for masonry structures, Ph.D. thesis, Delft University of Technology (1996).

[26] G. Milani, P. B. Lourenço, A. Tralli, Homogenization approach for the limit analysis of out-of-plane loaded masonry walls, Journal of Structural Engineering 132 (2006) 1650 - 1663.

[27] P. B. Lourenço, G. Milani, A. Trall, A. Zucchini, Analysis of masonry structures: review of recent trends in homogenization techniques, Canadian Journal of Civil Engineering 34 (2007) 1443 - 1457.

[28] R. de Borst, J. J. C. Remmers, A. Needleman, M. A. Abellan, Discrete vs smeared crack models for concrete fracture: Bridging the gap, International Journal for Numerical and Analytical Methods in Geomechanics 28 (2004) 583607.

[29] K. Meguro, H. Tagel-Din, Applied element method for structural analysis: Theory and application for linear materials, Journal of Earthquake Engineering, JSCE 17 (1) (2000) 21 - 35.

[30] H. Helmy, H. Hadhoud, S. Mourad, Infilled masonry walls contribution in mitigating progressive collapse of multistory reinforced concrete structures according to ufc guidelines, International Journal of Advanced Structural Engineering 7 (2015) $233-247$.

[31] A. Elshaer, H. Mostaga, H. Salem, Progressive collapse assessment of multistory reinforced concrete structures subjected to seismic actions, KCSE Journal of Engineering (2016) $1-11$.

[32] T. A. Rose, An approach to the evaluation of blast loads on finite and semi-infinite structures, Ph.D. thesis, Cranfield University (2001). 